\title{
Dotação Intelectual: Comparações entre Avaliação por Teste e Nomeação por Pais
}

\author{
Juliana Célia de Oliveiraํㅜ, Carolina Sertã Passos, Altemir José Gonçalves Barbosa \\ Universidade Federal de Juiz de Fora, Juiz de Fora-MG, Brasil
}

\section{RESUMO}

Este estudo teve como objetivo comparar dois tipos de medidas (Teste de Raciocínio e Nomeação por Pais) utilizadas na avaliação de dotação intelectual. Para tanto, aplicaram-se a Bateria de Provas de Raciocínio (BPR-5) e a versão para pais das Escalas de Sobre-Excitabilidade, mais especificamente a escala de Sobre-Excitabilidade Intelectual (SEI), em alunos do ensino fundamental $(n=67)$ e em seus pais ou responsáveis $(n=67)$, respectivamente. Ao comparar as duas medidas, observou-se que os estudantes que apresentaram melhor desempenho no escore geral da BPR-5 também tenderam a apresentar médias mais altas na SEI. Foram obtidas correlações positivas, significativas, que variaram de fracas a moderadas, entre a SEI, a capacidade cognitiva geral e alguns tipos de raciocínio (verbal, abstrato e numérico). A comparação e as associações positivas entre os dois instrumentos permitem sugerir que ambos investigam construtos correlatos. Entretanto, as medidas devem ser consideradas como complementares e não substitutivas.

Palavras-chave: sobre-excitabilidade; superdotação; avaliação psicológica.

ABSTRACT - Intellectual Giftedness: Comparisons between Assessment Testing and Nomination by the Parents

This study aimed to compare two types of measures (reasoning test and parent's nomination) used in the assessment of intellectual giftedness. Accordingly, the Reasoning Test Battery (BPR-5) and the parents version of the Overexcitability Scales, more specifically the Intellectual Overexcitability scale (SEI) were applied with elementary school students $(n=67)$ and their parents or guardians $(n=67)$, respectively. When comparing the two measures, it was observed that students who performed better in the BPR-5 also tended to receive higher means in the SEI. Significant positive correlations, which ranged from weak to moderate, were obtained between the SEI, the general reasoning ability, and some types of reasoning (verbal, abstract, and numeric). The comparison and positive associations between the two instruments indicate that both investigate related constructs. However, the measures should be considered as complementary rather than substitutive.

Keywords: overexcitability; giftedness; psychological evaluation.

\section{RESUMEN - Dotación Intelectual: Comparaciones entre Evaluación por Prueba y Nombramiento por Padres}

Este estudio tuvo como objetivo comparar dos tipos de medidas (prueba de raciocinio y nombramiento por padres) utilizadas en la evaluación de dotación intelectual. Razón por la cual, se aplicaron la Batería de Pruebas de Razonamiento (BPR-5) y la versión para padres de las Escalas de Sobreexcitabilidad, más específicamente se aplicó la Escala de Sobreexcitabilidad Intelectual (SEI) en alumnos de la enseñanza básica $(n=67)$ y en sus padres o responsables $(n=67)$. Al comparar las dos medidas, se observó que los estudiantes que presentan mejor desempeño en la BPR-5, también tienden en presentar medias más altas en la SEI. Se obtuvo correlaciones positivas, significativas y débiles entre la SEI y la capacidad general de razonamiento. La SEI también se correlacionó positiva y significativamente con algunos tipos de razonamiento (Verbal, Abstracto y Numérico). La comparación y las asociaciones positivas entre los dos instrumentos permiten sugerir que ambos investigan constructos relacionados. Con todo, las medidas deben ser consideradas como complementarias y no substitutivas. Palabras clave: sobreexcitabilidad; superdotación; evaluación psicológica.

A (super)dotação ${ }^{2}$ intelectual pode ser considerada uma "chave mestra" para vários talentos, especialmente na área acadêmica. Ela é inclusive considerada sinônimo de dotação em certos estados dos Estados Unidos
(McClain \& Pfeiffer, 2012). Historicamente, esse fato esteve atrelado à avaliação do quociente de inteligência (QI) e ainda, para alguns autores, possuir um QI igual ou superior a 120 tem sido considerado um dos critérios

Endereço para correspondência: Universidade Federal de Juiz de Fora, Instituto de Ciências Humanas, Campus Universitário São Pedro. Rua José Lourenço Kelmer, S/N, 36001-970, Juiz de Fora, MG. E-mail: julianaoliveirapsi@gmail.com Apoio: Monitoria UFJF. Agência Financiadora: FAPEMIG.

2 O Ministério da Educação do Brasil (2015) utiliza a expressão altas habilidades/superdotação para designar os alunos que "demonstram potencial elevado em qualquer uma das seguintes áreas, isoladas ou combinadas: intelectual, acadêmica, liderança, psicomotricidade e artes. E também apresentam elevada criatividade, grande envolvimento na aprendizagem e realização de tarefas em áreas de seu interesse". Como esta terminologia tem sido criticada (Güenther, 2006; Rangni \& Costa, 2011), optou-se por utilizar termo dotação para designar posse superior de uma capacidade (intelectual, motora, física, social etc.). 
para se definir dotação (Olszewski-Kubilius, Subotnik, \& Worrell, 2015).

Tanto no âmbito internacional (McClain \& Pfeiffer, 2012) quanto no nacional (Barbosa, Scheline, \& Almeida, 2012), os testes de inteligência, desempenho intelectual e raciocínio têm sido uma das principais - se não a principal - medidas de dotação em geral. Porém, desde a última década do século XX e na primeira década do século XXI, o uso desses instrumentos como principal estratégia para a avaliação de dotação passou a ser amplamente criticado (Freeman \& Güenther, 2000; Oliveira, 2007; OlszewskiKubilius, 2006). Uma das críticas diz respeito ao fato de se restringir o todo a uma parte, ou seja, entende-se dotação como capacidade intelectual, negligenciando-se outros domínios, tais como acadêmico e artístico (Carman, 2011). Além disso, mesmo que seja considerado somente o domínio intelectual, há limitações, pois, dentre outros aspectos, as potencialidades intelectuais extrapolam o QI (Virgolim, 2007).

Para enriquecer o processo de identificação de dotação em geral, a literatura tem destacado a necessidade de se utilizarem outros informantes (pais, professores e pares) e outras fontes de informação (escalas de autorrelato, produções etc.), além da avaliação de diferentes construtos que constituem o fenômeno (motivação, liderança, criatividade etc.), não restringindo a avaliação aos testes de inteligência ou raciocínio e às pessoas avaliadas (Li, Lee, Pfeiffer, \& Petscher, 2008; McClain \& Pfeiffer, 2012; Oliveira, 2007; Pereira, 2010). Isso diminuiria provavelmente o número de falsos negativos, especialmente aqueles oriundos de grupos marginalizados (Hertzog, 2009).

Dentre as diversas teorias que envolvem múltiplos informantes na identificação de dotação, destaca-se a perspectiva de Renzulli, que considera que entre 15 e $20 \%$ dos estudantes possuem essa característica (Renzulli, 1998). Para o processo de identificação, o autor propôs o Modelo das Portas Giratórias (Renzulli,1990), que estabelece uma série de passos que auxiliam a definir os alunos que irão compor o chamado "pool de talentos". No primeiro passo - nomeação por testes -, há a utilização de qualquer medida padronizada de avaliação intelectual (p. ex., teste de inteligência, aptidão, raciocínio e desempenho acadêmico), sendo considerado com dotação, o estudante que alcançar o percentil local igual ou superior a $92 \mathrm{em}$ pelo menos um subteste (Renzulli, 1990). Em média, se comparado aos outros passos, $50 \%$ dos alunos que irão compor o grupo de estudantes com dotação são selecionados nessa etapa.

A nomeação por pais pode ser utilizada em uma etapa intermediária do Modelo das Portas Giratórias, denominada Caminhos Alternativos. Diferente do primeiro passo, a nomeação parental não inclui automaticamente as pessoas no grupo de dotação, sendo necessário um estudo de caso para determinar se ocorrerá ou não a inserção dela no pool de discentes com talento (Renzulli,
1990). Destaca-se, entretanto, que essa estratégia tem sido pouco utilizada (Oliveira, 2007) e possui limitações (Freeman \& Güenther, 2000; Oliveira \& Anache, 2005), apesar de os pais possuírem informações importantes que não são geralmente obtidas por meio de testes (Gallegos, Álvarez-González, \& Costa, 2015). Dentre as limitações, destacam-se a tendência à sobrenomeação (Barbosa et al., 2008; Havigerová, Juklová, \& Haviger, 2013; Yewchuk \& Bibby, 1988), de indicarem mais meninos que meninas (Freeman \& Güenther, 2000) e de apresentarem concepções equivocadas, confusas ou estereotipadas de inteligência e dotação (Güenther, 2006; Oliveira \& Anache, 2005). Não obstante às tais restrições, a nomeação parental tem sido recomendada (Mönks, Van Boxtel, Roelofs, \& Sandres, 1986; Reichenberg \& Landau, 2009), especialmente no caso dos estudantes que não foram selecionados por meio de testes ou por nomeação por professores. Tem-se sugerido, também, que o desenvolvimento de escalas mais objetivas e válidas e uma preparação adequada dos pais podem tornar essa estratégia mais confiável (Oliveira, 2007).

Apesar das limitações e da carência de medidas com evidências de validade, os pais têm participado de processos de identificação de dotação, fornecendo informações sobre diferentes construtos relacionados à dotação (Barbosa et al., 2008). Dentre eles, este texto enfatiza a sobre-excitabilidade (SE), mais especificamente a sobre-excitabilidade intelectual (SEI).

A SE é um dos conceitos-chave da Teoria da Desintegração Positiva de Dabrowski (1964) que mais se destaca no campo de estudos sobre dotação (Ackerman, 2009). Trata-se de uma teoria que enfatiza o desenvolvimento socioemocional, sendo a SE um atributo importante da personalidade presente em muitas pessoas com dotação (Daniels \& Meckstroth, 2009).

A SE se refere à forma como alguns indivíduos, especialmente aqueles com dotação, tendem a reagir perante estímulos relacionados a um ou mais dos seguintes padrões: psicomotor, sensorial, imaginativo, intelectual e emocional (Dabrowski, 1972, 1996). Os indivíduos com maiores níveis dessa característica reagem aos diversos estímulos, externos e/ou internos, de forma intensificada, tanto qualitativamente quanto quantitativamente. A SE Intelectual é expressada por meio de uma busca intensa por conhecimento e soluções para os problemas. Indivíduos com esse padrão são, por exemplo, curiosos, questionadores, observadores, apresentam independência de pensamento, realizam análises teóricas e reflexões filosóficas e se destacam pela facilidade para formular respostas lógicas e novos conceitos (Dabrowski, 1972, 1996).

Diversos estudos têm demonstrado que pessoas com dotação tendem a apresentar níveis elevados de SE, em um ou mais padrões (p. ex., intelectual, emocional, sensorial, imaginativo e/ou psicomotor) (Alias, Rahman, Majid, \& Yassin, 2013; Al-Onizat, 2013; Carman, 2011; 
Siu, 2010). Entretanto, a SE Intelectual, mais especificamente, tem sido observada em indivíduos que foram identificados como possuidores de dotação intelectual apenas por testes (Siu, 2010; Van den Broeck, Hofmans, Cooremans, \& Staels, 2014; Wirthwein \& Rost, 2011) ou em processos com múltiplos informantes e múltiplas fontes de informação (Harrisson \& Haneghan, 2011; Tieso, 2007).

Grande parte dos instrumentos que avaliam as SEs faz uso de autorrelato. O Overexcitability Questionnaire Two (OEQ-II) (Falk, Lind, Miller, Piechowski, \& Silverman, 1999) é um exemplo típico desse tipo de medida e tem sido amplamente utilizado em estudos sobre dotação (AlOnizat, 2013; Chang \& Kuo, 2013; He \& Wong, 2014). Entretanto, quando se consideram os pais como fonte de informação, o número de instrumentos para essa finalidade é muito limitado. O Overexcitability Inventory for Parents (OIP), adaptado por Helen Dudeney (Institute for the Study of Advanced Development, 2007) a partir do OEQII, parece ser a única medida destinada a pais ou responsáveis. No entanto, não foram publicados, ainda, resultados referentes ao processo de obtenção de evidências de validade do OIP.

Se recorrer ao uso de testes é um procedimento comum na avaliação da dotação intelectual (Kaufman \& Sternberg, 2008), o número de estudos que tiveram os pais como informantes na avaliação desse construto é bastante reduzido. Quando se tratam de investigações que comparam testagem e nomeação por pais na avaliação de dotação intelectual, a escassez é, evidentemente, ainda maior. Além disso, os resultados obtidos pelos poucos estudos encontrados são controversos. Enquanto Olszewski-Kubilius (2006) verificou que o número de estudantes que foram identificados via nomeação parental não discrepou significativamente dos nomeados por testes, nos estudos de Barbosa et al. (2008), Havigerová et al., (2013) e Yewchuk e Bibby (1988) foi observada uma tendência de os pais nomearem mais estudantes que a testagem. Mesmo no caso dessas três pesquisas há discrepâncias nos resultados, pois, na primeira e na segunda, foram obtidas correlações positivas, significativas, fraca e moderada, respectivamente, entre as duas formas de nomeação e, na terceira, essa correlação não foi significativa.

É evidente, assim, a necessidade de estudos que comparem nomeação por teste e nomeação parental para identificar dotação intelectual. Mais necessárias ainda são as investigações que associem testagem de dotação intelectual e avaliação da SE Intelectual por parte de pais. Nesse sentido, o presente estudo teve como objetivo comparar dois tipos de medidas utilizadas na nomeação de dotação intelectual. Para a análise da dotação intelectual por meio de teste foi utilizada a Bateria de Provas de Raciocínio (BPR-5). Já para a nomeação parental, foi utilizada a versão para pais das Escalas de SobreExcitabilidade (ESE-Pais), mais especificamente a escala de Sobre-Excitabilidade Intelectual (SEI).

\section{Método}

\section{Participantes}

Participaram do estudo 67 estudantes do quinto ano do ensino fundamental de uma escola pública mineira, bem como seus pais, mães ou responsáveis $(N=67)$. A idade média em anos dos alunos foi de 10,46 $(D P=1,55)$ e $55,2 \%$ desses participantes $(n=37)$ eram do sexo feminino. Os alunos foram escolhidos intencionalmente por fazerem parte de uma instituição que estava realizando um processo de avaliação de dotação nos alunos e anualmente participava de um programa de identificação e desenvolvimento de talentos. Dentre outras formas de avaliação, a escola utiliza, no processo de identificação, múltiplas fontes de informação, tais como nomeação por pais, professores, medidas de raciocínio, criatividade e avaliação periódica. Não foi controlado quem preencheu o instrumento para pais, mães ou responsáveis, sendo solicitado apenas que o preenchimento fosse feito pelo cuidador que melhor conhecesse o aluno.

\section{Instrumentos}

Nos alunos, foi aplicada a forma A da BPR-5 (Almeida \& Primi, 2000). Trata-se de um instrumento de avaliação das habilidades cognitivas que oferece estimativas do funcionamento cognitivo geral, possibilitando, assim, a avaliação simultânea do fator $g$ e de fatores mais específicos. Essa forma é destinada a estudantes do ensino fundamental, e é composta por cinco provas: (a) raciocínio verbal (RV), que indica extensão do vocabulário e capacidade de estabelecer relações abstratas entre conceitos verbais; (b) raciocínio abstrato (RA), indicando a capacidade de estabelecer relações abstratas em situações novas para as quais se possui pouco conhecimento previamente aprendido; (c) raciocínio mecânico (RM), que avalia o conhecimento prático de mecânica e física; (d) raciocínio espacial (RE), que indica a capacidade em formar representações mentais e manipulá-las, transformando-as em novas representações; e (e) raciocínio numérico ( $\mathrm{RN}$ ), indicando a capacidade de raciocínio com símbolos numéricos em problemas quantitativos e conhecimento de operações aritméticas básicas. O cálculo referente ao escore geral da BPR-5 pode ser obtido de duas formas: pela soma dos resultados brutos nas cinco provas, resultando no Eg-5; e pela soma dos resultados brutos de quatro provas (RA, RN, RV e RE), representado pelo Eg-4. Destaca-se que a BPR-5 é um instrumento recomendado pelo Sistema de Avaliação de Testes Psicológicos (Conselho Federal de Psicologia, 2018) e que diversas evidências de validade e fidedignidade têm sido observadas na medida (Almeida \& Primi, 2000).

Os pais, mães ou responsáveis preencheram a versão para pais das ESE-Pais (Barbosa \& Oliveira, 2013). O instrumento é composto por 55 itens, subdivididos em cinco padrões de SE - Sensorial, Psicomotor, Imaginativo, Intelectual e Emocional - e distribuídos aleatoriamente. 
Para o presente estudo, foram utilizados somente os dados referentes à SEI da ESE-Pais. Esse padrão é representado por 10 itens, cada um contendo uma afirmação que denota uma característica-chave da SE Intelectual. Para o preenchimento da escala, é solicitado aos participantes que indiquem o quanto cada afirmação se parece com o(a) aluno(a) alvo. Isso é feito em uma escala Likert de cinco pontos, que varia entre $0-\mathrm{Nada}$ a ver com o(a) estudante - a 4 - Tudo a ver com o(a) estudante. O escore do padrão é calculado a partir da média dos seus itens. Deve-se mencionar que, até o momento, foram obtidas evidências de validade e fidedignidade somente da versão para os estudantes das ESE, sendo observadas propriedades psicométricas adequadas para as escalas (Oliveira \& Barbosa, 2015; Oliveira \& Barbosa, 2018).

\section{Procedimento}

O presente estudo foi aprovado em um Comitê de Ética em Pesquisa e seguiu os quesitos éticos necessários. A ESE-Pais foi enviada pelos estudantes aos seus responsáveis. Foram anexadas instruções ao instrumento, apresentando os objetivos do estudo e o preenchimento das escalas, bem como um Termo de Consentimento Livre e Esclarecido. Após a autorização e o preenchimento da medida, as ESE-Pais foram entregues aos pesquisadores pelos próprios estudantes.

A BPR-5 foi aplicada em sala de aula coletivamente, mas com preenchimento individual, após ser obtido o consentimento da direção da escola e dos pais e ser assinado o termo de assentimento pelos próprios estudantes para a participação no estudo. A aplicação das cinco provas foi dividida em duas etapas, ocorridas em dias diferentes: a primeira se destinou ao preenchimento das provas RV, RA e RM; e a segunda às provas RE e RN. O procedimento de aplicação seguiu as normas estabelecidas no manual do instrumento. A pontuação do teste também seguiu o manual, mas não foi utilizada a norma nacional para aferir os percentis, sendo utilizado o próprio grupo como parâmetro para tanto (norma local).

\section{Análise de Dados}

$\mathrm{Na}$ análise de dados, foi adotado o percentil local igual ou superior a 92 - ponto de corte para nomeação por testes sugerido por Renzulli (1990) - para compor os grupos de estudantes que se destacaram na BPR-5. O alfa de Cronbach foi utilizado com a finalidade verificar a consistência interna da SEI. Para a associação entre os instrumentos, utilizou-se o coeficiente de correlação de Pearson. Realizou-se, também, o teste t para comparar as médias dos escores da SEI entre os grupos com percentil maior e menor que 92 obtidos na BPR-5. O tamanho do efeito $(d)$ de Cohen (1988) foi obtido nos casos em que foram encontradas diferenças significativas entre os grupos comparados e os seguintes valores de referências foram considerados: 0,20 (pequeno), 0,50 (médio) ou 0,80 (grande).

\section{Resultados}

Os valores de média (M) e de desvio padrão (DP) para o total da amostra nos instrumentos utilizados são apresentados na Tabela 1. Nela, também são indicados os valores máximo e mínimo (mín-máx) obtidos em cada prova.

Tabela 1

Valores Mínimos e Máximos, Médias e Desuios Padrão da ESE-Pais e da BPR-5

\begin{tabular}{cccccc}
\hline Variáveis & Mín-Máx & $\begin{array}{c}M(D P) \\
\text { Amostra geral }\end{array}$ & $\begin{array}{c}M(D P) \\
\text { Masculino }\end{array}$ & $\begin{array}{c}M(D P) \\
\text { Feminino }\end{array}$ & Comparações intergrupos \\
\hline SEI & $0,70-4,00$ & $2,87(0,64)$ & $3,04(0,77)$ & $2,74(0,47)$ & $t(65,67)=1,87 ; p=0,07$ \\
RV & $2-17$ & $9,97(3,98)$ & $10,43(4,15)$ & $9,59(3,85)$ & $t(65,67)=0,85 ; p=0,39$ \\
RA & $1-22$ & $13,91(4,53)$ & $13,63(4,78)$ & $14,13(4,37)$ & $t(65,67)=-, 44 ; p=0,65$ \\
RM & $2-16$ & $6,63(2,61)$ & $6,83(2,36)$ & $6,46(2,81)$ & $t(65,67)=0,58 ; p=0,56$ \\
RE & $0-18$ & $9,19(3,95)$ & $9,90(3,66)$ & $8,62(4,14)$ & $t(65,67)=1,32 ; p=0,19$ \\
RN & $0-12$ & $6,66(3,54)$ & $6,83(3,56)$ & $6,51(3,57)$ & $t(65,67)=0,36 ; p=0,72$ \\
Eg-5 & $15-73$ & $46,36(12,46)$ & $47,63(12,40)$ & $45,32(12,60)$ & $t(65,67)=0,75 ; p=0,45$ \\
Eg-4 & $9-63$ & $40,80(12,38)$ & $38,86(11,92)$ & $39,73(12,07)$ & $t(65,67)=0,65 ; p=0,52$ \\
\hline
\end{tabular}

Ao relacionar os escores médios obtidos na SEI e as médias de acertos nas provas da BPR-5 ao sexo dos estudantes, não foram observadas associações significativas. Os resultados obtidos nas comparações intergrupos também são apresentados na Tabela 1 . A confiabilidade da medida da SEI na ESE-Pais pode ser considerada elevada. $\mathrm{O}$ alfa de Cronbach dela foi de 0,81 .
Ao analisar os escores obtidos na BPR-5, observou-se que $35,82 \%$ dos estudantes $(n=24)$ atingiram o percentil $92 \mathrm{em}$ pelo menos uma prova. Dentre eles, 16 alunos $(66,66 \%)$ obtiveram desempenho nesse ponto de corte em apenas uma prova; seis $(25 \%)$ atingiram essa pontuação em duas provas; um $(4,17 \%)$ em três provas; e um $(4,17 \%)$ em quatro provas. Ao considerar o escore 
geral fornecido pela bateria, constatou-se que sete estudantes $(10,45 \%)$ alcançaram o percentil 92 tanto em Eg-5 quanto em Eg-4.

Quando associados os escores obtidos na BPR-5 e na SEI, observaram-se correlações positivas significativas com magnitudes que variaram entre fracas a moderadas entre a ESE intelectual e três provas de raciocínio: RV, RA e RN. Associações positivas, significativas e fracas também foram observadas ao correlacionar a SE Intelectual ao Eg-5 e ao Eg-4. A Tabela 2 apresenta os valores das correlações e os níveis de significância, obtidas pela correlação de Pearson.

Tabela 2

Correlações entre o Padrão de SE da ESE-Pais e as Provas de Raciocínio da BPR-5

\begin{tabular}{cccccccc}
\hline \multicolumn{1}{c}{ ESE-Pais } & \multicolumn{7}{c}{ BPR-5 } \\
\hline \multirow{2}{*}{ SE Intelectual } & RA & RV & RM & RE & RN & Eg-5 & Eg-4 \\
& $0,25^{*}$ & $0,47^{* *}$ & 0,11 & 0,09 & $0,34^{* *}$ & $0,39^{* *}$ & $0,38^{* *}$ \\
\hline
\end{tabular}

${ }^{*} p<0,05 ;{ }^{* *} p<0,01$

Após a subdivisão dos grupos com percentis acima e abaixo de 92, realizou-se um teste t para amostras independentes, a fim de associar os escores médios da ESEPais aos dois grupos em questão. Diferenças significativas foram observadas nos grupos quanto às provas RM e RN e quanto ao Eg-5 e Eg-4. Nesses casos, os estudantes que estavam no grupo de desempenho mais elevado apresentaram, na nomeação por pais, médias mais altas de SE Intelectual. O tamanho do efeito entre os grupos que tiveram diferenças significativas foi considerado entre médio e grande, obtendo os seguintes valores: RM $(d=0,83), \mathrm{RN}(1,00), \mathrm{Eg}-5(0,60)$ e Eg-4 $(0,87)$. A Tabela 3 apresenta as médias obtidas na ESE-Pais nos diferentes grupos. Nela é possível observar também que, apesar de em três provas não terem sido observadas diferenças significativas, as médias de SE Intelectual mostraram-se mais elevadas no grupo de alunos com escores mais altos nas provas RV, RA e RE.

Tabela 3

Médias da ESE-Pais nos Grupos por Percentis e Comparações Intergrupos

\begin{tabular}{|c|c|c|c|c|}
\hline & \multirow{2}{*}{ BPR-5 } & \multicolumn{2}{|c|}{ ESE-Pais } & \multirow{2}{*}{ Comparações intergrupos } \\
\hline & & $M$ & $D P$ & \\
\hline \multirow{2}{*}{ RV } & Percentil $\geq 92$ & 3,10 & 0,24 & \multirow{2}{*}{$t(65,67)=-0,82 ; p=0,42$} \\
\hline & Percentil $<92$ & 2,86 & 0,66 & \\
\hline \multirow{2}{*}{ RA } & Percentil $\geq 92$ & 3,05 & 0,60 & \multirow{2}{*}{$t(65,67)=-1,05 ; p=0,30$} \\
\hline & Percentil $<92$ & 2,84 & 0,64 & \\
\hline \multirow{2}{*}{$\mathrm{RM}$} & Percentil $\geq 92$ & 3,33 & 0,59 & \multirow{2}{*}{$t(65,67)=-2,03 ; p<0,05$} \\
\hline & Percentil $<92$ & 2,82 & 0,63 & \\
\hline \multirow{2}{*}{$\mathrm{RE}$} & Percentil $\geq 92$ & 3,03 & 0,67 & \multirow{2}{*}{$t(65,67)=-0,63 ; p=0,53$} \\
\hline & Percentil < 92 & 2,86 & 0,64 & \\
\hline \multirow{2}{*}{$\mathrm{RN}$} & Percentil $\geq 92$ & 3,42 & 0,63 & \multirow{2}{*}{$t(65,67)=-2,03 ; p<0,05$} \\
\hline & Percentil $<92$ & 2,83 & 0,55 & \\
\hline \multirow{2}{*}{ Eg-5 } & Percentil $\geq 92$ & 3,33 & 0,53 & \multirow{2}{*}{$t(65,67)=-2,03 ; p<0,05$} \\
\hline & Percentil $<92$ & 2,82 & 0,63 & \\
\hline \multirow{2}{*}{ Eg-4 } & Percentil $\geq 92$ & 3,33 & 0,53 & \multirow{2}{*}{$t(65,67)=-2,03 ; p<0,05$} \\
\hline & Percentil $<92$ & 2,82 & 0,63 & \\
\hline
\end{tabular}




\section{Discussão}

O presente estudo teve como objetivo comparar dois tipos de medidas utilizadas na nomeação de dotação intelectual. Utilizando-se a BPR-5, os resultados revelaram que mais de um terço dos alunos participantes apresentou desempenho igual ou superior ao percentil 92 na nomeação por testes em pelo menos uma das provas de raciocínio. Esse número está bem acima do estimado pelo Modelo das Portas Giratórias (Renzulli, 1990), que propõe que, em geral, até $20 \%$ dos estudantes deveriam compor o pool de discentes com dotação. Provavelmente, a quantidade de alunos se elevaria ainda mais se fossem utilizadas fontes distintas de informação, tais como professores e pares, e se fossem levadas em consideração outras áreas nas quais os alunos poderiam se destacar, como criatividade.

A proposta de Renzuli (1990) ainda prevê que, somente na etapa de nomeação por testes, aproximadamente $50 \%$ dos estudantes que comporiam o grupo com dotação seriam identificados. Caso isso se concretizasse, apenas sete alunos $(10,45 \%)$ da amostra pesquisada deveriam ter apresentado desempenho superior na BPR5 . Se fosse considerado somente o escore geral do instrumento, a porcentagem de estudantes que atingiram o percentil adotado se adequaria à proporção estimada. Entretanto, esse procedimento contraria a própria sugestão do autor (Renzulli, 1990), isto é, de que sejam utilizados como indicadores de dotação tanto os resultados gerais em um teste quanto os escores dos subtestes. $\mathrm{O}$ fato de a BPR-5 apresentar cinco provas que avaliam raciocínios específicos e, portanto, habilidades diferentes eleva o percentual indicado.

Reitera-se que, do mesmo modo que no presente estudo, algumas pesquisas tiveram como objetivo associar medidas de nomeação por pais e testes. Barbosa et al. (2008), por exemplo, verificaram correlação positiva, significativa e fraca entre os escores de nomeação parental para capacidades intelectuais e a testagem desse aspecto efetuada com o Teste de Raven (Raven, 2003) em estudantes de uma cidade brasileira. A mesma magnitude e sentido das correlações foram encontrados por Havigerová et al. (2013) ao comparar os escores da nomeação por pais com as pontuações obtidas no WISC III por estudantes da República Tcheca. Apesar dessas investigações se basearem em modelos teóricos distintos de inteligência e de utilizarem medidas diferentes na sua avaliação, os resultados de ambas pesquisas se assemelham aos da presente investigação, já que, ao associar a SEI aos tipos de raciocínios e ao escore geral da BPR-5, também foram observadas correlações fracas ou moderadas, positivas e significativas.

As semelhanças nos construtos e a associação positiva e significativa dos dois instrumentos utilizados no presente estudo permitem sugerir que ambos investigam construtos correlatos, mesmo que essa afirmação caiba somente para Eg-5, Eg-4, e algumas provas (RV, RA e RN). Reitera-se que a BPR-5 investiga raciocínios fortemente associados ao fator $\mathrm{g}$ e habilidades mais específicas e as provas RV, RA e RN envolvem a utilização de analogias e buscam investigar a relação existente entre palavras, figuras geométricas e números, respectivamente (Almeida \& Primi, 2000). Já a SE Intelectual diz respeito às características de personalidade que se expressam pela aceleração e intensificação das atividades mentais (Daniels \& Meckstroth, 2009) e se manifestam, por exemplo, por meio de questionamentos, curiosidades, buscas ativas por conhecimentos e soluções de problemas (Dabrowski, 1996). Nesse sentido, a magnitude limitada das correlações entre os instrumentos utilizados era esperada, tendo em vista que SE Intelectual e raciocínio não são construtos equivalentes.

Duas provas da BPR-5 - RM e RE - não se correlacionaram de forma significativa com a SEI da ESEPais. A avaliação do RM é composta por problemas práticos que envolvem conteúdos físico-mecânicos e na prova de RE é apresentada uma série de cubos tridimensionais com o objetivo de formar representações mentais, manipulá-las e transformá-las em novas representações (Almeida \& Primi, 2000). O construto intelectual investigado de forma mais prática nessas avaliações parece se diferir do avaliado na medida de relato dos pais, tendo em vista que a SEI analisa características mais abstratas da personalidade. Isso deve ser alvo de investigações adicionais, com amostras mais amplas, por exemplo, com análises de variáveis, que não foram investigadas no presente estudo, tais como características demográficas.

A literatura tem sugerido que meninos tendem a apresentar pontuações mais altas nas provas RM, RE e RN da BPR-5 que as meninas (Almeida \& Primi, 2000), bem como no padrão de SE Intelectual mensurado em diferentes instrumentos (Miller, Falk, \& Huang, 2009; Piirto \& Fraas, 2012; Van den Broeck et al., 2014). Entretanto isso não foi observado no presente estudo. Os resultados discrepantes podem estar relacionados às questões históricas e/ou culturais. No caso da BPR-5, os estudos de validação datam de quase duas décadas, podendo haver mudanças nos últimos anos no que se refere a padrões de estímulos relacionados aos diversos raciocínios. Já para a SE Intelectual, destaca-se que o estudo apresentado por Oliveira e Barbosa (2018) no contexto brasileiro, utilizando uma medida de SE para estudantes, também não observou a diferença entre os sexos quanto a esse padrão, sugerindo que meninas tendem a apresentar características de curiosidade, interesse por aprender assuntos novos, entre outros aspectos da SE Intelectual tanto quanto meninos.

O fato de as médias de SEI serem mais altas nos grupos de estudantes que apresentaram melhor desempenho nos escores geral da BPR-5 e em duas provas específicas (RM e RN), evidencia que, em grande 
parte, os alunos que se destacaram pela alta SEI também apresentaram elevada capacidade de raciocínio. Esses resultados corroboram os de outros estudos, que também observaram que a SEI se manifesta mais em indivíduos que foram identificados por meio de testes (Van den Broeck et al., 2014; Siu, 2010; Wirthwein \& Rost, 2011). Entretanto, ao considerar as demais provas (RV, RA e RE), é possível notar estudantes que, apesar de apresentarem alto nível do padrão de SE Intelectual não se destacaram nas provas específicas de raciocínio e vice-versa. Assim, pode-se observar certa independência entre raciocínio e SE e parece sensato afirmar que a SEI medida pela ESE-Pais e a BPR-5 não são medidas substitutivas e sim complementares. Sugere-se que algumas características avaliadas na SEI não são alvo das provas de raciocínio, mas que podem promover o desenvolvimento dessa função cognitiva.

Destaca-se que os resultados do presente estudo devem ser considerados com cautela, já que ele possui limitações em sua validade interna e externa. No primeiro caso, destacam-se as poucas evidências de validade da ESE-Pais. No presente estudo, foi possível observar que o padrão intelectual mensurado pela escala possui elevada consistência interna. Além disso, ao associá-la com instrumentos que investigam construtos correlatos (BPR-5), foi possível verificar boas evidências de validade baseadas na relação com variáveis externas. Fazem-se necessários, entretanto, estudos adicionais, que investiguem, por exemplo, as evidências de validade de critério e baseadas na estrutura interna.

No que se refere às limitações relacionadas à validade externa, destacam-se as caraterísticas amostrais, já que a amostra é pequena e composta somente por alunos do ensino fundamental de apenas uma escola pública. Tais limitações demandam cautela na generalização dos resultados. São necessários, portanto, estudos adicionais que contem com amostras mais amplas e diversificadas, de diferentes instituições, a fim de aumentar a representatividade da população investigada.

Não obstante as circunscrições metodológicas mencionadas, os resultados do presente estudo contribuem para a área, pois, ao observar certa independência entre as medidas, evidenciou a necessidade de se utilizar medidas distintas, que se complementam, como uma forma de superar as limitações dos processos tradicionais de identificação de dotação intelectual. São apresentados, ademais, resultados que constituem evidências de validade e fidedignidade para uma das escalas de instrumentos para pais - a SEI das ESE-Pais.

A nomeação por pais é um caminho alternativo e importante, devendo ser considerada em conjunto com outros instrumentos padronizados e outras informações advindas de múltiplas fontes, incluindo pares e professores. No presente estudo, os pais e/ou responsáveis foram informados sobre os objetivos da avaliação e orientados sobre como preencher o instrumento. Ressalta-se, contudo, a necessidade de orientar o sujeito fonte de informação sobre o construto SE por se tratar de um tema ainda pouco conhecido no Brasil, para que ele não incorra no erro de ser um "sobre-nomeador" ou "sub-nomeador".

Apesar das críticas e limitações da nomeação parental (Oliveira \& Anache, 2005; Güenther, 2006), não se deve desconsiderar que os pais constituem uma das influências primordiais no desenvolvimento cognitivo dos filhos (Reichenberg \& Landau, 2009) e, portanto, podem constituir uma fonte rica de informações sobre as capacidades intelectuais deles. Nota-se, desse modo, a necessidade de dispensar maior atenção a esse procedimento, especialmente no caso de estudantes que não foram selecionados por meio de testes. Além disso, a SEI pode ser uma via de acesso para uma identificação mais abrangente, possibilitando avaliar fatores relevantes que não são apreendidos, geralmente, por testes utilizados pela psicometria tradicional.

Sugere-se, ademais, como enfatizado por Renzulli (1990), que programas de busca ativa por estudantes com dotação nas escolas deve ir além do uso de testes, mais especificamente testes de inteligência. O pool de identificados não deve ser composto apenas por estudantes que se sobressaem em tarefas que exigem altas habilidades acadêmicas (domínios de aptidão acadêmica específica) ou elevada capacidade intelectual, mas também por aqueles que se destacam em aspectos que não são, necessariamente, avaliados por testes, como pensamento criativo-produtivo, capacidade de liderança, habilidade especial para artes, dentre outros domínios propostos pelo Ministério da Educação (Brasil, 2015). Evidencia-se, desse modo, a necessidade de que esses programas priorizem avaliações multidimensionais, combinando-as com informações de desenvolvimento, produtos, realizações etc.

Salienta-se, desse modo e por fim, a necessidade de, no Brasil, realizar mais estudos sobre identificação ativa de dotação intelectual em ambientes escolares. Ainda que as leis brasileiras assegurem a identificação e o desenvolvimento aos estudantes com dotação (Brasil, 2015), são poucos os serviços de atendimento voltados para esse grupo de pessoas no país (Passos \& Barbosa, 2017). Há que se contar, também, com um amplo leque de instrumentos, como a ESE-Pais e a BPR-5, que possuam evidências de validade e fidedignidade e que permitam a realização de uma avaliação psicoeducacional baseada em múltiplas fontes de informação (testes de inteligência, nomeação parental etc.) e em múltiplos informantes (pais, professores, estudantes etc.). Essa identificação é fundamental para tornar o processo que almeja transformar dotação em talentos mais confiável; algo que tem sido negligenciado no país. 


\section{Referências}

Ackerman, C. M. (2009). The essential elements of Dabrowski's theory of positive disintegration and how they are connected. Roeper Review, 31(2), 81-95. doi: 10.1080/02783199709553835

Alias, A., Rahman, S., Majid, R. A., \& Yassin, S. F. M. (2013). Dabrowski’s overexcitabilities profile among gifted students. Asian Social Science, 9(16), 120-125. doi: 10.5539/ass.v9n16p120

Almeida, L. S., \& Primi, R. (2000). Baterias de Provas de Raciocínio - BPR-5. São Paulo: Casa do Psicólogo.

Al-Onizat, S. H. (2013). The psychometric properties of a Jordanian version of Overexcitability Questionnaire-Two, OEQII. Criative Education, 4(1), 49-61. doi: 10.4236/ce.2013.41008

Brasil, Ministério da Educação. (2015). Orientações para implementação da política de educação especial na perspectiva da educação inclusiva. Recuperado de http:// portal.mec.gov.br/index.php?option=com_docman\&view=download\&alias=17237-secadi-documento-subsidiario-2015\&Itemid =30192

Barbosa, A. J. G., Pereira, C. E. S., Gonçalves, F. C., Passos, C. S., Miranda, O. B., Jesus, T. L., Silva, J. D., \& Moreira, P. S. (2008). Identificação de sobredotação intelectual: Uso de testes e nomeação parental. Em XIII Conferência Internacional de Avaliação Psicológica: Formas e Contextos, Braga.

Barbosa, A. J. G., Schelini, P. W., \&Almeida, L. C. (2012). Medidas de dotação e talento: Produção científica em psicologia (2006-2011). Em E. Boruchovitch, A. A. A. Santos; E. Nascimento (Eds.). Avaliação psicológica nos contextos educativo e psicossocial (pp. 33-52). São Paulo: Casa do Psicólogo.

Barbosa, A. J. G., \& Oliveira, J. C. (2013). Escalas de sobre-excitabilidade: Versão para pais. Juiz de Fora: Autor.

Carman, C. A. (2011). Adding personality to gifted identification: Relationships among traditional and personality-based constructs. Journal of Advanced Academics, 22(3), 412-446. doi: 10.1177/1932202X1102200303

Chang, H. J., \& Kuo, C. C. (2013). Overexcitabilities: Empirical studies and application. Learning and Individual Differences, 23 (2013), 53-63. doi: 10.1016/j.lindif.2012.10.010

Cohen, J. (1988). Statistical power for behavioral sciences. New York: Academic Press.

Conselho Federal de Psicologia, CFP (2018). Sistema de avaliação de testes psicológicos: Testes psicológicos aprovados para uso. Brasilia: Conselho Federal de Psicologia. Recuperado de http://satepsi.cfp.org.br/testesFavoraveis.cfm

Dabrowski, K. (1964). Positive disintegration. Boston: Little Brown.

Dabrowski, K. (1972). Psychoneurosis is not an illness: Neuroses and psychoneuroses from the perspective of positive disintegration. London: Gryf.

Dabrowski, K. (1996). Multilevelness of emotional and instinctive functions. Lublin, Poland: Towarzystwo Naukowe Katolickiego Uniwersytetu Lubelskiego.

Daniels, S., \& Meckstroth, E. (2009). Nurturing the sensitivity, intensity and developmental potential of youg gifted children. Em S. Daniels; M. M. Piechowski (Eds.). Living with intensity (pp. 33-56). Scottsdale, AZ: Great Potential Press.

Freeman, J., \& Güenther, Z. C. (2000). Educando os mais capazes: Ideias e ações comprovadas. São Paulo: EPU.

Falk, R. F., Lind, S., Miller, N. B., Piechowski, M. M., \& Silverman, L. K. (1999). The Overexcitability Questionnaire Two (OEQ II). Manual, scoring system, and questionnaire. Denver: Institute for the Study of Advanced Development.

Gallegos, S. L. V., Álvarez-González, B., \& Costa, M. B. P. (2015). Los padres también cuentan. Validación del cuestionario "Conociendo a tu hijo, tu hija”: identificación de niño(a)s de 9 a 10 años con altas capacidades. Ensaio: Avaliação e Políticas Públicas em Educação, 23(89), 795-820. doi: 10.1590/S0104-40362015000400001

Güenther, Z. (2006). Capacidade e talento: Um programa para a escola. São Paulo: EPU.

Harrison, G. E., \& Haneghan, J. P. V. (2011). The gifted and the shadow of the night: Dabrowski's overexcitabilities and their correlation to insomnia, death anxiety, and fear of the unknown. Journal for the Education of the Gifted, 34(4), 669-697. doi: 10.1177/016235321103400407

Havigerová,J., Juklová, K., \& Haviger, J. (2013). When parents and teachers assess intellectual giftedness of preschool children. New Educational Review, 34(4),188-197. Recuperado de https://www.researchgate.net/profile/Mihaela_Brumen/publication/263540387_Attitudes_of_ Parents_toward_Learning_Foreign_Languages_-_a_Slovene_Case_Study/links/541691920cf2bb7347db4d18.pdf\# page=188

He, W., \& Wong, W. (2014). Greater male variability in overexcitabilities: Domain-specific patterns. Personality and Individual Differences, 66(2014), 27-32. doi: 10.1016/j.paid.2014.03.002

Hertzog, N. B. (2009). The arbitrary nature of giftedness. Em L. V. Shavinina (Eds.). International handbook on giftedness (pp. 205-215). Heidelberg, Germany: Springer.

Institute for the Study of Advanced Development. (2007). Overexcitability Inventory for Parents (adaptad by H. Dudeney). Denver, CO: Author.

Kaufman, S. B., \& Sternberg, R. J. (2008). Conceptions of giftedness. Em S. I. Pfeiffer (Ed.), Handbook of giftedness in children (pp. 71-91). Tallahassee, FL: Springer.

Li, H., Lee, D., Pfeiffer, S. I., \& Petscher, Y. (2008). Parent ratings using the Gifted Rating Scales - School Form: Reliability and validity for Chinese students. Educational and Psychological Measurement, 68(4), 659-675. doi: 10.1177/0013164407313365

McClain, M., \& Pfeiffer, S. (2012). Identification of gifted students in the united states today: A look at state definitions, policies, and practices. Journal of Applied School Psychology, 28(1), 59-88. doi: 10.1080/15377903.2012.643757

Miller, N. B., Falk, R. F., \& Huang, Y. (2009). Gender identity and the overexcitability profiles of gifted college students. Roeper Review, 31(3), 161-169. doi: 10.1080/02783190902993920

Mönks, F. J., Van Boxtel, H. W., Roelofs, J. J. W., \& Sandres, M. P. M. (1986). The identification of gifted children in secondary education and a description of their situation in Holland. Em K. Heller; J. F. Feldhusen (Eds.). Identifying and nurturing the gifted: an international perspective (pp. 39-66). New York: Hans Huber Publishers/Hogrefe International.

Olszewski-Kubilius, P. (2006). Comparisons between talent search students qualifying via scores on standardized tests and via parent nomination. (Talent Search Qualifying). Roeper Review, 28(3), 157-166. doi: 10.1080/02783190609554355

Olszewski-Kubilius, P., Subotnik, R. F., \& Worrell, F. C. (2015). Conceptualizations of giftedness and the development of talent: implications for counselors. Journal of Counseling \& Development, 93(2), 143-152. doi: 10.1002/j.1556-6676.2015.00190.x

Oliveira, E. P. L. (2007). Alunos sobredotados: a aceleração escolar como resposta educativa. (Tese de doutorado), Programa de Pós-graduação em Psicologia da Educação, Universidade do Minho, Braga, Portugal. Recuperado de http://repositorium.sdum.uminho.pt

Oliveira, C. G., \& Anache, A. A. (2005). A identificação e o encaminhamento dos alunos com altas habilidades/superdotação em Campo Grande - MS. Revista Educação Especial, 27. Recuperado de http://coralx.ufsm.br/revce/ceesp 
Oliveira, J. C., \& Barbosa, A. J. G. B. (2015). Escalas de Sobre-excitabilidade: Construção e evidências de validade baseadas no conteúdo e na estrutura interna. Psicologia: Reflexão e Crítica, 28(4), 639-648. doi: 10.1590/1678-7153.201528404

Oliveira, J. C., \& Barbosa, A. J. G. (2018). Invariância fatorial e normatização das Escalas de Sobre-Excitabilidade. Psico-USF, 23(4), 751-762. doi: 10.1590/1413-82712018230414

Passos, C. S., \& Barbosa, A. J. G. (2017). Desenvolvimento de talentos no Brasil: Uma análise dos serviços de atendimento. Educação em Revista, 33(2017), e143211. doi: 10.1590/0102-4698143211

Pereira, C. E. S. (2010). Identificação de estudantes talentosos: Uma comparação entre as perspectivas de Renzulli e Güenther (Dissertação de mestrado em Psicologia). Programa de Pós-Graduação em Psicologia, Universidade Federal de Juiz de Fora, Juiz de Fora.

Piirto, J., \& Fraas, J. (2012). A mixed-methods comparison of vocational and identified-gifted high school students on the overexcitability questionnaire. Journal for the Education of the Gifted, 35(1), 3-34. doi: 10.1177/0162353211433792

Raven, J. C. (2003). Matrizes progressivas: Escala geral, séries A, B, C, D e E. Rio de Janeiro: CEPA.

Rangni, R. A., \& Costa, M. P. R. (2011). Altas habilidades/superdotação: Entre termos e linguagens. Revista Educação Especial, $24(41)$, 467-482. doi: 10.5902/1984686X3056

Reichenberg, A., \& Landau, E. (2009). Families of gifted children. Em L. V. Shavinina (Ed.), International Handbook on Giftedness (pp. 873883). Heidelberg, Germany: Springer.

Renzulli, J. S. (1990). A practical system for identifying gifted and talented students. Early Childhood Development, 63(1), 9-18. doi: 10.1080/0300443900630103

Renzulli, J. S. (1998). The three-ring conception of giftedness. Em S. M. Baum, S. M. Reis \& L. R. Maxfield (Eds.). Nurturing the gifts and talents of primary grade students (pp. 50-72). Mansfield Center, CT: Creative Learning Press.

Siu, A. F. Y. (2010). Comparing overexcitabilities of gifted and non-gifted school children in Hong Kong: Does culture make a difference? Asia Pacific Journal of Education, 30(1), 71-83. doi: 10.1080/02188790903503601

Tieso, C. L. (2007). Overexcitabilities: A new way to think about talent? Roeper Review, 29(4), 232-239. doi: 10.1080/02783190709554417

Van den Broeck, W. V., Hofmans, J., Cooremans, S., \& Staels, E. (2014). Factorial validity and measurement invariance across intelligence levels and gender of the Overexcitabilities Questionnaire-II (OEQ-II). Psychological Assessment, 26(1), 55-68. doi: 10.1037/a0034475

Virgolim, A. M. R. (2007). Altas habilidades e desenvolvimento intelectual. Em D. S. Fleith \& E. M. L. S. Alencar (Eds.). Desenvolvimento de talentos e altas habilidades: Orientação a pais e professores (pp. 25-50). Porto Alegre: Artmed.

Wirthwein, L., \& Rost, D. H. (2011). Focussing on overexcitabilities: Studies with intellectually gifted and academically talented adults. Personality and Individual Differences, 51(3), 337-342. doi: 10.1016/j.paid.2011.03.041

Yewchuk, C., \& Bibby, M. A. (1988). A comparison of parent and teacher nomination of gifted hearing-impaired students. American Annals of the Deaf, 133(5), 344-348. doi: 10.1353/aad.2012.0725

\section{Sobre os autores}

Juliana Célia de Oliveira é psicóloga, mestre e doutora em Psicologia pela Universidade Federal de Juiz de Fora. Atualmente é Professora no curso de Psicologia da Faculdade Machado Sobrinho e na Faculdade Metodista Granbery.

Carolina Sertã Passos é psicóloga, mestre em Psicologia pela Universidade Federal de Juiz de Fora. Atualmente, é psicóloga da Prefeitura de Santo Antônio do Aventureiro/MG.

Altemir José Gonçalves Barbosa é psicólogo, mestre e doutor em Psicologia pela Pontifícia Universidade Católica de Campinas. Atualmente é Professor do Departamento de Psicologia e do Programa de Pós-graduação Stricto Sensu em Psicologia da Universidade Federal de Juiz de Fora. 